\title{
Unconventional molecular replacement for helical transmembrane proteins using AMPLE
}

Felix Simkovic $^{1}$, Jens Thomas $^{1}$, Olga Mayans ${ }^{2}$, Daniel Rigden ${ }^{1}$

${ }^{1}$ Department Of Biochemistry, University Of Liverpool, Liverpool, United Kingdom, ${ }^{2}$ Department of Biology, Universität Konstanz, Konstanz, Germany

E-mail: hlfsimko@liv.ac.uk

Transmembrane helical proteins are underrepresented in the PDB due to the experimental difficulties associated with their protein expression and crystallisation. This results in a small pool of structures being available for conventional homologybased Molecular Replacement (MR) and places a premium on the development of unconventional homology-independent approaches.

AMPLE is a pipeline for using unconventional models in automated MR. It has been particularly successful in its cluster-andtruncate approach to creating ensemble search models from ab initio molecular models for globular targets [1]. Recent work has focussed on exploring the viability of AMPLE's approach to transmembrane protein targets. By sampling multiple clusters of ab initio models and using new scoring algorithms, we have been able to double the success rate of AMPLE on a difficult set of test cases without an increase in processing time. Additionally, exploring new bioinformatics approaches, such as restraining $a b$ initio structure prediction using predicted residue-residue contacts, has pushed the boundaries of AMPLE's success [2].

Combining the latest developments, we have been exploring the effectiveness of AMPLE on a selection of alpha-helical transmembrane proteins. Our work has demonstrated that AMPLE is a powerful method to use on this class of proteins. AMPLE outperforms a baseline approach based on short alpha-helices and yields structure solutions from search models in which the modelling correctly predicted irregular features in the target (see figure, where the search model is blue and the target green). AMPLE is able to solve the majority of cases in our test set with an average runtime of less than two days on a single processor.

[1] Bibby, J. et al. (2012). Acta Cryst. D68, 1622-1631.

[2] Simkovic, F. et al. (2016). IUCr] 3 (Pt 4), 259-270.

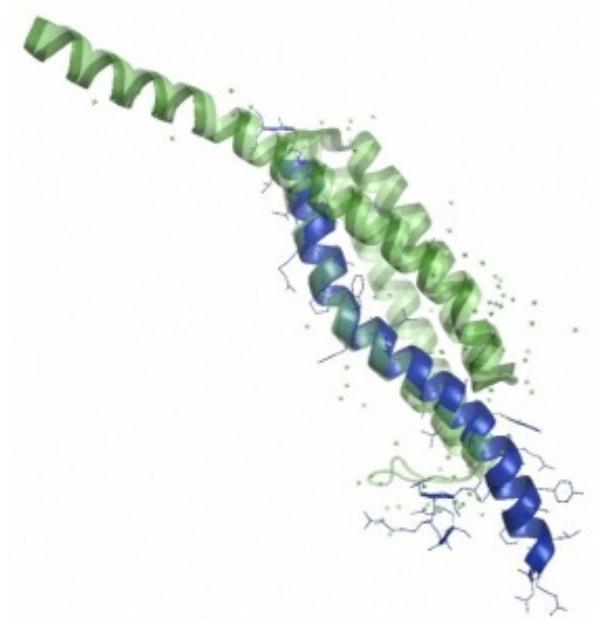

Keywords: Automated Molecular Replacement, Helical Transmembrane Proteins, AMPLE 